\title{
Clinical, serological and echocardiographic examination of healthy field dogs before and after vaccination with a commercial tetravalent leptospirosis vaccine
}

Andrea M. Spiri ${ }^{1,2}$, Sabrina Rodriguez-Campos ${ }^{4}$, José M. Matos ${ }^{3}$, Tony M. Glaus ${ }^{3}$, Barbara Riond ${ }^{1}$, Claudia E. Reusch ${ }^{3}$, Regina Hofmann-Lehmann ${ }^{1,2}$ and Barbara Willi ${ }^{1,3^{*}}$

\begin{abstract}
Background: Leptospirosis is a re-emerging bacterial zoonosis caused by spirochetes of the genus Leptospira. Severe disease has been reported in dogs in Europe despite vaccination with bivalent Leptospira vaccines. Recently, a tetravalent canine Leptospira vaccine (Nobivac ${ }^{\oplus}$ L4) was licenced in Europe. The goal of this study was to investigate clinical signs, microscopic agglutination test (MAT) titres, haematology, blood biochemistry, cardiac (c) Troponin I levels and echocardiography before and after vaccination with this tetravalent vaccine. Forty-eight healthy dogs were prospectively enrolled and vaccinated twice, 3-4 weeks apart (T0 and T1). Before vaccination (T0) and 16-31 days after the second vaccination (T2), MAT $(n=48)$, haematology $(n=48)$, blood biochemistry $(n=36)$ and cTroponin I measurements $(n=29)$ were performed, and MAT was repeated 347-413 days after the second vaccination $(T 3, n=44)$. Echocardiography was performed before the first and second vaccination (T0 and $\mathrm{T} 1, n=24)$.
\end{abstract}

Results: Mild and transient clinical signs within 5 days following the first and second vaccination occurred in 23\% and $10 \%$ of the dogs, respectively. Before the first vaccination (TO), all dogs showed negative MAT titres for the tested serovars except for Canicola (50\% with titres 100-400). At T2, positive MAT titres to the serovars Canicola (100\%), Australis (89\%), Grippotyphosa (86\%), Bratislava (60\%), Autumnalis (58\%), Copenhageni (42\%), Pomona $(12 \%)$, Pyrogenes (8\%) and Icterohaemorrhagiae (2\%) were found. Median to high titres $(\geq 400)$ were most common to the serovar Canicola (92\%) and less common to the serovars Australis (41\%), Grippotyphosa (21\%), Bratislava (12\%), Autumnalis (4\%), Pyrogenes (4\%) and Pomona (2\%). At T3, positive MAT titres (titre range: 100400 ) were found in $2-18 \%$ of the dogs to serovars of the vaccine serogroups and in $2-18 \%$ of the dogs to the non-vaccine serovars Pomona, Autumnalis, Pyrogenes and Ballum. Haematology, blood biochemistry, cTroponin I levels and echocardiography results did not change significantly following vaccination.

Conclusions: Clinical signs following vaccination with Nobivac ${ }^{\circledR} L 4$ were transient and mild in all cases. Seroconversion differed considerably among individual dogs and among the vaccine serogroups.

Keywords: Nobivac ${ }^{\oplus}$ Lepto 6, Leptospira, Tetravalent vaccine, Vaccination, Seroconversion, Microscopic agglutination test, Adverse events

\footnotetext{
* Correspondence: bwilli@vetclinics.uzh.ch

${ }^{1}$ Clinical Laboratory, Vetsuisse Faculty, University of Zurich, Winterthurerstr.

260, 8057 Zurich, Switzerland

${ }^{3}$ Clinic for Small Animal Internal Medicine, Vetsuisse Faculty, University of

Zurich, Winterthurerstr. 260, 8057 Zurich, Switzerland

Full list of author information is available at the end of the article
} 


\section{Background}

Leptospirosis is a re-emerging bacterial zoonosis of global importance caused by spirochetes of the genus Leptospira [1]. Dogs can be infected with a wide range of pathogenic Leptospira serovars and shed the leptospires in their urine during acute infection [2]. Leptospires can survive for months in water and moist soil [3], and indirect transmission through contact with contaminated soil or water is thought to play an important role in the epidemiology of leptospirosis.

In Europe, canine leptospirosis in dogs has gained increasing attention in the last several years [4]. Following infection with pathogenic leptospires, dogs can develop a severe, multi-systemic disease associated with high mortality [2]. Besides this severe course of infection, mild clinical signs and asymptomatic infections have been documented. Acute leptospirosis in dogs is mainly associated with acute kidney or liver injury and respiratory signs associated with pulmonary haemorrhage [5]. Furthermore, myocarditis has been documented in humans with leptospirosis and has also been suspected in infected dogs $[6,7]$. The clinical manifestation of leptospirosis depends not only on the causative serovar but also on host-specific factors such as age and the immune status of the dog [8].

The most commonly used diagnostic test for leptospirosis in dogs is the demonstration of antibodies in the microscopic agglutination test (MAT). Serial dilutions of patient sera are thereby incubated with a selection of pathogenic Leptospira serovars, and agglutination of the organisms is assessed by darkfield microscopy. Crossreactivity among serovars only allows for a serogroupspecific diagnosis, and positive titres following vaccination, previous exposure and during chronic asymptomatic infection complicate the interpretation of the results [2]. Generally, the serovar that gives the highest titre is regarded as the infecting serogroup or serovar, although this diagnosis is, at best, presumptive [9]. Post-vaccination titres have been assessed in small groups of dogs following vaccination with bivalent and tetravalent vaccines [10-12]. These studies revealed that titres usually decline by 4 months after vaccination, although persistently high titres have been documented which have been explained by natural exposure to Leptospira field serovars [10]. PCR can be used as an alternative diagnostic method to detect leptospiral DNA in biological samples such as blood, urine or tissue. However, leptospires can only transiently be detected in the blood and urine of infected animals [13], and antibiotic therapy is thought to clear bacteraemia and leptospiruria [14]. The detection of the agents in biological samples by bacterial culture is a definitive proof of infection [2], but leptospires are fastidious organisms requiring special culture media, and they grow very slowly after 2 to up to
24 weeks in culture, making this diagnostic procedure impractical in a clinical setting.

Despite the introduction of efficient bivalent Leptospira bacterin vaccines some decades ago [15], clinical leptospirosis has recently been documented among numerous vaccinated dogs in Europe [16]. The currently licenced bivalent Leptospira vaccines in Europe contain serovars belonging to the serogroups Canicola and Icterohaemorrhagiae. These vaccines have been shown to prevent leptospirosis caused by serovars belonging to these two serogroups, but the vaccines induce only partial or no immunity to heterologous serogroups [17-19]. Canicola and Icterohaemorrhagiae were previously thought to be the main serogroups causing clinical leptospirosis in dogs. Recent seroepidemiological studies revealed, however, that dogs are susceptible to a broad range of serovars. In Europe, antibodies against the serogroups Icterohaemorrhagiae, Canicola, Grippotyphosa, Pomona, Australis and Sejroe have most commonly been documented in clinically healthy and diseased dogs [4].

In 2012, a new canine tetravalent inactivated Leptospira ${\text { vaccine }\left(\text { Nobivac }^{\oplus} \text { L4, in Switzerland licenced as Nobivac }\right.}^{\odot}$ Lepto 6, MSD Animal Health GmbH, Luzern, Switzerland) that contains antigens from serovars from four different serogroups, including Canicola, Icterohaemorrhagiae, Australis and Grippotyphosa was licenced in Europe $[20,21]$. Because serology represents the mainstay in the diagnosis of leptospirosis in dogs, the serological response following vaccination can interfere with the interpretation of MAT results in suspect clinical cases [22]. So far, no data on the serological response following vaccination with this novel tetravalent vaccine have been published. Furthermore, recent pharmacovigilance reports from Germany and Switzerland suggest that adverse events after vaccination with Leptospira vaccines have significantly increased since 2013 and 2014, respectively, following the introduction of novel, multivalent Leptospira vaccines in these countries $[23,24]$. The authors of the reports speculated that the increasing number of Leptospira serovars included in these vaccines could result in higher antigenicity of the vaccines. However, studies investigating the incidence of adverse events following the vaccination of dogs did not reveal a significant increase in hypersensitivity reactions following Leptospira vaccination when compared with other vaccines $[25,26]$. Finally, there have been recent anecdotal reports describing cardiac abnormalities suspicious of acute myocarditis after vaccination (S. Jenni, personal communication). Specifically, at the second vaccination, new heart murmurs had been auscultated that had not been present at the first vaccination. Subsequent echocardiographic examinations reportedly had revealed left ventricular dilation and hypocontractility with mitral annulus dilation and associated mitral insufficiency as the explanation for the murmur. 
The goal of the present study was to evaluate the serological response of dogs after vaccination with a commercially available, inactivated, tetravalent Leptospira vaccine and to investigate whether the dogs showed clinical signs and alterations in haematology, blood biochemistry and echocardiography following vaccination.

\section{Methods}

\section{Study design, sample and data collection and sample processing}

This monocentric, longitudinal, prospective study was performed at the Clinic for Small Animal Internal Medicine, University of Zurich, between August 2013 and May 2015. Dogs were prospectively enrolled within the study when they met the following inclusion criteria: older than 8 weeks of age, judged clinically healthy by the owner, in good general health according to clinical examination, no vaccination within 4 weeks and no medical therapy within 2 months prior to study enrolment, with the exception of antiparasitic treatment. The study design and number of dogs that received each procedure are shown in Table 1. The day of the first vaccination was defined as T0. All dogs were vaccinated twice with a commercial tetravalent leptospirosis vaccine (Nobivac ${ }^{\oplus}$ L4, in Switzerland licenced as Nobivac ${ }^{\bullet}$ Lepto 6, MSD Animal Health GmbH) 34 weeks apart (T0 and T1). Additional vaccine components licenced for concurrent administration were applied as required (Table 1). Blood samples were collected by venipuncture before the first (T0) and second vaccination (T1) and 41-54 days (T2) and 368-434 days after the first vaccination (T3). Blood samples were processed or frozen at $-80{ }^{\circ} \mathrm{C}$ within $4 \mathrm{~h}$ of collection. A medical history and clinical examination were performed at each presentation by a veterinary surgeon (AS) or a boardcertified internal medicine specialist (BW). Echocardiography was performed before the first (T0) and second vaccination (T1) by board-certified cardiologists (JM, TG) at the division of cardiology, Vetsuisse Faculty, University of Zurich. Adverse vaccine events were suspected when clinical signs occurred within 5 days of vaccination and belonged to one of the following groups: lethargy or inappetence, local reaction at the injection site (signs of tenderness or pain, cutaneous swelling or mass, lameness in a limb close to the injection site), non-specific signs (periuria, diarrhoea, vomiting) or a hypersensitivity reaction (swelling of the face, hives or bumps on the face or body, hypersalivation, tachypnoea, or respiratory distress). Data on adverse vaccine events were collected from the medical history and clinical examination performed at each presentation.

\section{Haematology, blood biochemistry and cTroponin I}

Haematology and blood biochemistry tests and measurements of cTroponin I were performed at the Clinical Laboratory, Vetsuisse Faculty, University of Zurich. Haematology was performed from EDTA anticoagulated whole blood within $4 \mathrm{~h}$ of sample collection. Blood biochemistry was determined batch-wise from serum samples stored at $-80{ }^{\circ} \mathrm{C}$. Haematology and blood biochemistry were run on a Sysmex XT-2000iV (Sysmex Corporation, Kobe, Japan) [27] and Cobas Integra 800 instrument (Roche Diagnostics AG, Rotkreuz, Switzerland), respectively. The clinical chemistry parameters included: albumin, total protein, alanine aminotransferase (ALAT), alkaline phosphatase (AP), bilirubin, urea, creatinine and lipase. The laboratory's device-specific reference intervals were applied. cTroponin I was measured on an Immulite 1000 (Siemens Healthcare Diagnostics GmbH, Zurich, Switzerland). The serum cTroponin I concentration was measured using a commercially available assay that had been validated for the dog [28]. The test's lower limit of detection was $0.2 \mathrm{ng} / \mathrm{mL}$, and cTroponin I values exceeding $0.2 \mathrm{ng} / \mathrm{mL}$ were considered increased.

Table 1 Study design and number of dogs that received each procedure

\begin{tabular}{|c|c|c|c|c|}
\hline Time point & TO & T1 & $\mathrm{T} 2$ & T3 \\
\hline Days after first presentation & 0 & $20-28$ & $41-54$ & $368-434$ \\
\hline \multicolumn{5}{|l|}{ Procedure/number of dogs } \\
\hline Vaccination & 48 & 48 & - & 44 \\
\hline Clinical examination & 48 & 48 & 48 & 44 \\
\hline Echocardiography & 24 & 24 & - & - \\
\hline MAT & 48 & - & 48 & 44 \\
\hline Haematology & 48 & - & 48 & - \\
\hline Blood biochemistry & 36 & - & 36 & - \\
\hline cTroponin I & 29 & - & 29 & - \\
\hline \multicolumn{5}{|c|}{ Number of dogs receiving other vaccine components } \\
\hline Nobivac ${ }^{\oplus}$ DHPPi & 16 & 3 & - & - \\
\hline Nobivac ${ }^{\oplus} \mathrm{Pi}$ & 10 & 1 & - & - \\
\hline
\end{tabular}




\section{Microscopic agglutination test}

The MAT was performed at the National Reference Laboratory for animal leptospirosis at the Institute of Veterinary Bacteriology, Vetsuisse Faculty, University of Bern, Switzerland. The MAT was performed batch-wise from serum samples stored at $-80{ }^{\circ} \mathrm{C}$ that were sent by priority mail at room temperature to the laboratory. Samples were examined for the presence of antibodies against a standard panel of 14 pathogenic Leptospira strains commonly used in Switzerland (Table 2) by MAT according to Office International des Epizooties (OIE) standards [29]. To prevent inter-observer variability, all samples were tested by the same person. The end-point titre refers to the reciprocal of the highest serum dilution at which at least $50 \%$ agglutination occurs. In this study, sera with a titre of 100 , indicating past exposure to Leptospira [8], were considered seropositive.

\section{Echocardiography}

Echocardiographic examinations were performed with continuous ECG monitoring on laterally recumbent unsedated dogs using a GE Vivid 7 ultrasound unit (Vivid 7, GE Medical Systems, Munich, Germany). Standard two-dimensional, M-mode and colour Doppler echocardiographic views were obtained from standard right parasternal views using a $7 \mathrm{MHz}$ transducer. The primary focus was qualitative left ventricular size and function as well as valvular integrity and function. Quantitated parameters were left ventricular diameter in diastole and systole (LVDd and LVDs, respectively), the shortening fraction (\%FS) from M-Mode images, and the left atrial to aortic ratio (LA/Ao) in short axis [30, 31].

\section{Statistical analyses}

Statistical analyses were performed using Analyse-it ${ }^{\bullet}$ for Microsoft Excel version 1.0.5.0 (Analyse-it Software, Ltd., Leeds, United Kingdom) and GraphPad Prism 5.03 (GraphPad Software, Inc., CA, USA). The Wilcoxon signed rank test $\left(\mathrm{p}_{\mathrm{w}}\right)$ was used to compare the results from the same dog at two different time points. Correlation between MAT titres and time elapsed since the last Leptospira vaccination was assessed using the Spearman rank correlation test (correlation coefficient: $r_{\mathrm{sp}}$ ). Proportions between groups were analysed with the Fisher's exact test $\left(\mathrm{p}_{\mathrm{F}}\right) . P$-values $<0.05$ were considered significant.

\section{Results}

\section{Characteristics of the study population}

A total of 48 dogs were included in the study. The characteristics of the study population are shown in Table 3. All but one dog had previously received a Leptospira vaccine; all vaccine products were inactivated bivalent Leptospira vaccines containing serovars from the serogroups Canicola and Icterohaemorrhagiae. The vaccines belonged to several different brands (Canigen ${ }^{\circ}$ L $(n=28)$, Canigen $7(n=1)$, Virbac, Glattbrugg, Switzerland; Canimed ${ }^{\circ} \mathrm{L}(n=1)$, Eurican ${ }^{\circ} \mathrm{L}(n=1)$, Merial, Lyon, France; Nobivac ${ }^{\circ}$ Lepto $(n=8)$, Vetamun ${ }^{\circ}$ Lepto $(n=6)$, MSD Animal Health GmbH; Vanguard ${ }^{\circ}$ $7(n=2)$, Zoetis Schweiz GmbH, Zurich, Switzerland). All 48 dogs completed the primary vaccination schedule, and 44 dogs were sampled after 1 year (T3, Table 1); the remaining four dogs died within 1 year after vaccination for unrelated reasons $(n=2)$, moved to another country $(n=1)$ or did not keep the appointment after

Table 2 The 14 Leptospira spp. strains used as live antigens in the microscopic agglutination test ${ }^{a}$

\begin{tabular}{|c|c|c|c|}
\hline Genomospecies & Serogroup & Serovar & Strain \\
\hline \multirow[t]{10}{*}{ Leptospira interrogans } & Australis & Australis & Ballico \\
\hline & Australis & Bratislava & Jez-Bratislava \\
\hline & Autumnalis & Autumnalis & Akiyami \\
\hline & Bataviae & Bataviae & Swart \\
\hline & Canicola & Canicola & Hond Utrecht IV \\
\hline & Icterohaemorrhagiae & Icterohaemorrhagiae & RGA \\
\hline & Icterohaemorrhagiae & Copenhageni & M20 \\
\hline & Pomona & Pomona & Pomona \\
\hline & Pyrogenes & Pyrogenes & Salinem \\
\hline & Sejroe & Hardjo & Hardjoprajitno \\
\hline \multirow[t]{3}{*}{ Leptospira borgpetersenii } & Ballum & Ballum & Mus127 \\
\hline & Tarassovi & Tarassovi & Perepelitsin \\
\hline & Sejroe & Sejroe & M84 \\
\hline Leptospira kirschneri & Grippotyphosa & Grippotyphosa & Moskva V \\
\hline
\end{tabular}

${ }^{2}$ All reference strains were obtained from the Royal Tropical Institute (KIT), Amsterdam, The Netherlands 
Table 3 Characteristics of the 48 dogs of this study

\begin{tabular}{|c|c|c|c|c|}
\hline Parameter & Number of dogs & Range & Median & $95 \% \mathrm{Cl}^{\mathrm{a}}$ \\
\hline Age (years) & 48 & $0.4-14$ & 5 & $4.2-6.3$ \\
\hline Body weight (kg) & 48 & $5.5-51.2$ & 21 & $18.0-25.1$ \\
\hline Days to prior leptospirosis vaccination & 47 & $31-1745$ & 379 & $386.5-640.5$ \\
\hline Parameter & Number of dogs (\%) & & & \\
\hline \multicolumn{5}{|l|}{ Gender } \\
\hline Female intact & $16(33)$ & & & \\
\hline Female castrated & $16(33)$ & & & \\
\hline Male intact & $14(29)$ & & & \\
\hline Male castrated & $2(4)$ & & & \\
\hline \multicolumn{5}{|l|}{ Pedigree } \\
\hline Yes & $39(81)$ & & & \\
\hline No & $9(19)$ & & & \\
\hline \multicolumn{5}{|l|}{ Breed } \\
\hline Barzoi & $8(17)$ & & & \\
\hline Beagle & $4(8)$ & & & \\
\hline Labrador Retriever & $5(10)$ & & & \\
\hline Jack Russel Terrier & $2(4)$ & & & \\
\hline Rhodesian Ridgeback & $2(4)$ & & & \\
\hline Shetland Sheepdog & $6(13)$ & & & \\
\hline Malinois & $2(4)$ & & & \\
\hline Whippet & $2(4)$ & & & \\
\hline Other breeds ${ }^{\mathrm{b}}$ & $8(17)$ & & & \\
\hline
\end{tabular}

${ }^{\mathrm{a}} \mathrm{Cl}$, confidence interval

bother breeds comprised: Old German Shepherd Dog, Australian Kelpie, Bavarian Mountain Hound, Golden Retriever, German Wirehaired Pointer, Small Munsterlander, Poitevin, Schipperke (each $n=1$ )

1 year $(n=1)$. The two dogs that died were 11 and 14 years old and were euthanized because of histiocytic sarcoma or acute monoparesis that occurred 137 and 340 days after the second vaccination, respectively.

\section{Clinical examination}

Clinical examination was unremarkable in $75-90 \%$ of the dogs at each presentation (Additional file 1). Clinical findings included vaginal discharge due to juvenile vaginitis or heat, conjunctivitis, faint heart murmurs, splenomegaly, focal pyoderma, inflammation or fistula in the anal region, red and scaly ears, fluid-filled bowel loops, enlarged prostate and depigmentation of the nose.

Following the first and second vaccination, adverse vaccine events were reported by the owners of 11 (23\%) and $5(10 \%)$ dogs, respectively (Additional file 1). The clinical signs occurred within 5 days of vaccination and were transient and mild in all cases. The clinical signs mainly included local reactions at the injection site, lethargy or inappetence. Three dogs showed a single episode of vomiting or periuria. A total of 6 out of 11 dogs with adverse events after the first vaccination had received additional vaccine components (Nobivac ${ }^{\circ} \mathrm{DHPPi}$, $n=3$, Nobivac ${ }^{\circ} \mathrm{Pi}, n=3$ ). None of the five dogs with adverse events after the second vaccination had received additional vaccine components. The proportion of animals that showed adverse vaccine events was not significantly different between dogs that had or had not received additional vaccine components $\left(\mathrm{p}_{\mathrm{F}}=1.0\right)$. Other anamnestic abnormalities that occurred after 5 days of vaccination were reported in $5(10 \%)$ and $4(8 \%)$ dogs after the first (T0) and second vaccination (T1), respectively. They included a single episode of vomiting or diarrhoea 10-14 days after vaccination, a depigmentation of the planum nasale that started 7 days after vaccination, acute trembling and a focal epileptic seizure 3 and 4 weeks after vaccination, respectively, coughing 3 weeks after vaccination, a reduced general condition 10 days after vaccination and tenesmus due to prostate enlargement 3 weeks after vaccination. At the time of the 1-year booster vaccination (T3), two dogs had died (see above), one dog had developed laryngeal paralysis, one dog suffered from idiopathic epilepsy and one dog had developed and recovered from an acute encephalitis. 


\section{Microscopic agglutination test}

MAT results for the 14 tested serovars are shown in Table 4 for the serogroups included in the vaccine and in Table 5 for the serogroups that are not included in the vaccine. At first presentation and before the first vaccination (T0), all dogs had negative MAT titres $(<100)$ for all tested serovars, except for the serovar Canicola, for which $50 \%$ of the dogs showed titres $\geq 100$ at T0 (Table 4). All of the seropositive dogs had been previously vaccinated with a vaccine that contained a serovar of the serogroup Canicola. In the 20 dogs with titres of 100, the previous Leptospira vaccine had been given 101-1745 days prior to study enrolment. In the three dogs with titres of 200 , the leptospirosis vaccine had been given 176-554 days prior to study enrolment, and in the one dog with a titre of 400 , the vaccine had been given 129 days before enrolment. There was no significant correlation between the MAT titres to the serovar Canicola and the time elapsed since the last vaccination $\left(\mathrm{r}_{\mathrm{sp}}=-0.006\right)$.

Positive MAT titres $(\geq 100)$ measured after the second vaccination (T2) were most common for the serovar Canicola (100\%), followed by the serovars Australis (89\%), Grippotyphosa (86\%), Bratislava (60\%), Autumnalis (58\%), Copenhageni (42\%), Pomona (12\%), Pyrogenes (8\%) and Icterohaemorrhagiae (2\%, Tables 4 and 5$)$. Median to high titres $(\geq 400)$ were most commonly found for the serovar Canicola (92\%), followed by the serovars Australis (41\%), Grippotyphosa (21\%), Bratislava (12\%), Autumnalis (4\%), Pyrogenes (4\%) and Pomona (2\%); none of the dogs developed titres $\geq 400$ for the serovars Copenhageni or Icterohaemorrhagiae. Maximal MAT titres measured at T2 for the serovars were as follows: Canicola (3200), Australis (3200), Grippotyphosa (800), Bratislava (800), Autumnalis (3200), Copenhageni (200), Pomona (400), Pyrogenes (800) and Icterohaemorrhagiae (100).
At the 1-year booster appointment (T3), $2-18 \%$ of the dogs showed positive MAT titres to serovars of the vaccine serogroups, and the titres were generally low $(<400$, Table 4). A total of $2-18 \%$ of the dogs showed positive MAT titres to the non-vaccine serovars Pomona, Autumnalis, Pyrogenes and Ballum (Table 5). The seven dogs with an MAT titre of 400 against the serovar Pyrogenes at T3 had negative titres against the other tested serovars at this time point and had also been seronegative for the serovar Pyrogenes following the second vaccination (T2). Positive MAT titres were not detected at any time point during the study for $L$. interrogans serovar Bataviae or for $L$. borgpetersenii serovars Tarassovi, Hardjo and Sejroe (data not shown).

\section{Haematology, blood biochemistry and cTroponin I}

The haematological parameters determined before (T0) and after the second vaccination (T2) revealed no significant differences, with the exception of a slight but significant increase in haematocrit $\left(\mathrm{p}_{\mathrm{w}}=0.02\right)$ and a decrease in total monocyte count at $\mathrm{T} 2$ compared to $\mathrm{T} 0\left(\mathrm{p}_{\mathrm{W}}=0.04\right.$; Additional file 2). For the blood biochemistry results, significant differences between $\mathrm{T} 0$ and $\mathrm{T} 2$ were only evident for the AP activity (decrease; $\mathrm{p}_{\mathrm{W}}=0.04$ ) and the urea concentration (increase; $\mathrm{p}_{\mathbb{W}}=0.03$; Additional file 3). These differences were small, and the median AP activity and urea concentration remained within the reference range at both time points. The cTroponin I concentrations were obtained in 29 dogs. At T0, cTroponin I concentrations were below the detection limit in 27 dogs; two dogs (both Barzois) had measurable cTroponin I levels of 0.39 and $0.50 \mathrm{ng} / \mathrm{mL}$. At T2, cTroponin I concentrations were below the detection limit in 27 dogs; two dogs (both Barzois) had measurable cTroponin I levels of 0.23 and $0.29 \mathrm{ng} / \mathrm{mL}$; both dogs had had undetectable values at T0. There was no significant difference between the cTroponin I levels at T0 and T2.

Table 4 Microscopic agglutination test results for the vaccine serogroups before and after vaccination

\begin{tabular}{|c|c|c|c|c|c|c|c|c|c|c|c|c|c|c|c|c|c|c|}
\hline Species & \multicolumn{3}{|c|}{ L. interrogans } & \multicolumn{3}{|c|}{ L. interrogans } & \multicolumn{3}{|c|}{ L. interrogans } & \multicolumn{3}{|c|}{ L. interrogans } & \multicolumn{3}{|c|}{ L. interrogans } & \multicolumn{3}{|c|}{ L. kirschneri } \\
\hline Serogroup & \multicolumn{3}{|c|}{ Canicola } & \multicolumn{3}{|c|}{ Icterohaemorrhagiae } & \multicolumn{3}{|c|}{ Icterohaemorrhagiae } & \multicolumn{3}{|c|}{ Australis } & \multicolumn{3}{|c|}{ Australis } & \multicolumn{3}{|c|}{ Grippotyphosa } \\
\hline Serovar & \multicolumn{3}{|c|}{ Canicola } & \multicolumn{3}{|c|}{ Copenhageni } & \multicolumn{3}{|c|}{ Icterohaemorrhagiae } & \multicolumn{3}{|c|}{ Bratislava } & \multicolumn{3}{|c|}{ Australis } & \multicolumn{3}{|c|}{ Grippotyphosa } \\
\hline Time point & T0 & $\mathrm{T} 2$ & $\mathrm{~T} 3$ & TO & $\mathrm{T} 2$ & $\mathrm{~T} 3$ & TO & $\mathrm{T} 2$ & $\mathrm{~T} 3$ & T0 & $\mathrm{T} 2$ & T3 & TO & $\mathrm{T} 2$ & T3 & TO & $\mathrm{T} 2$ & $\mathrm{~T} 3$ \\
\hline \multicolumn{19}{|c|}{ Percentage of dogs with titre } \\
\hline$<100$ & 50 & 0 & 91 & 100 & 58 & 95 & 100 & 98 & 98 & 100 & 40 & 98 & 100 & 11 & 82 & 100 & 14 & 95 \\
\hline 100 & 42 & 2 & 9 & 0 & 33 & 5 & 0 & 2 & 2 & 0 & 19 & 0 & 0 & 27 & 11 & 0 & 42 & 5 \\
\hline 200 & 6 & 6 & 0 & 0 & 9 & 0 & 0 & 0 & 0 & 0 & 29 & 2 & 0 & 21 & 7 & 0 & 23 & 0 \\
\hline 400 & 2 & 34 & 0 & 0 & 0 & 0 & 0 & 0 & 0 & 0 & 10 & 0 & 0 & 25 & 0 & 0 & 19 & 0 \\
\hline 800 & 0 & 29 & 0 & 0 & 0 & 0 & 0 & 0 & 0 & 0 & 2 & 0 & 0 & 8 & 0 & 0 & 2 & 0 \\
\hline 1600 & 0 & 27 & 0 & 0 & 0 & 0 & 0 & 0 & 0 & 0 & 0 & 0 & 0 & 6 & 0 & 0 & 0 & 0 \\
\hline 3200 & 0 & 2 & 0 & 0 & 0 & 0 & 0 & 0 & 0 & 0 & 0 & 0 & 0 & 2 & 0 & 0 & 0 & 0 \\
\hline Total number of dogs & 48 & 48 & 44 & 48 & 48 & 44 & 48 & 48 & 44 & 48 & 48 & 44 & 48 & 48 & 44 & 48 & 48 & 44 \\
\hline
\end{tabular}

Positive titres are shown in bold 
Table 5 Microscopic agglutination test results for selected non-vaccine serogroups before and after vaccination

\begin{tabular}{|c|c|c|c|c|c|c|c|c|c|c|c|c|}
\hline Species & \multicolumn{3}{|c|}{ L. interrogans } & \multicolumn{3}{|c|}{ L. interrogans } & \multicolumn{3}{|c|}{ L. interrogans } & \multicolumn{3}{|c|}{ L. borgpetersenii } \\
\hline Serogroup & \multicolumn{3}{|c|}{ Pomona } & \multicolumn{3}{|c|}{ Autumnalis } & \multicolumn{3}{|c|}{ Pyrogenes } & \multicolumn{3}{|c|}{ Ballum } \\
\hline Serovar & \multicolumn{3}{|c|}{ Pomona } & \multicolumn{3}{|c|}{ Autumnalis } & \multicolumn{3}{|c|}{ Pyrogenes } & \multicolumn{3}{|c|}{ Ballum } \\
\hline Time point & TO & $\mathrm{T} 2$ & T3 & TO & $\mathrm{T} 2$ & T3 & T0 & $\mathrm{T} 2$ & T3 & T0 & $\mathrm{T} 2$ & T3 \\
\hline \multicolumn{13}{|c|}{ Percentage of dogs with titre } \\
\hline$<100$ & 100 & 88 & 87 & 100 & 42 & 95 & 100 & 92 & 82 & 100 & 100 & 98 \\
\hline 100 & 0 & 8 & 9 & 0 & 50 & 5 & 0 & 4 & 2 & 0 & 0 & 2 \\
\hline 200 & 0 & 2 & 2 & 0 & 4 & 0 & 0 & 0 & 0 & 0 & 0 & 0 \\
\hline 400 & 0 & 2 & 2 & 0 & 2 & 0 & 0 & 2 & 16 & 0 & 0 & 0 \\
\hline 800 & 0 & 0 & 0 & 0 & 0 & 0 & 0 & 2 & 0 & 0 & 0 & 0 \\
\hline 1600 & 0 & 0 & 0 & 0 & 0 & 0 & 0 & 0 & 0 & 0 & 0 & 0 \\
\hline 3200 & 0 & 0 & 0 & 0 & 2 & 0 & 0 & 0 & 0 & 0 & 0 & 0 \\
\hline Total number of dogs & 48 & 48 & 44 & 48 & 48 & 44 & 48 & 48 & 44 & 48 & 48 & 44 \\
\hline
\end{tabular}

Positive titres are shown in bold

\section{Echocardiography}

Left ventricular (LVDd) and left atrial (LA/Ao) size did not significantly change between the first (T0) and the second (T1) vaccination, and mitral insufficiency could not be documented in any dog. Additionally, systolic function, as assessed by LVDs and \%FS, did not change significantly between $\mathrm{T} 0$ and $\mathrm{T} 1$. Looking at individual dogs, there was not any dog that showed a notable increase in LVDd or LVDs. In none of the dogs could any arrhythmia be recognized at any time point during the study.

\section{Discussion}

This is the first independent study evaluating the serological and clinical response in field dogs following vaccination with a novel tetravalent Leptospira vaccine. Our results suggest that the vaccine was generally well tolerated and that there was marked variation among individual dogs and among different vaccine serovars regarding the extent of seroconversion following vaccination.

Up to $23 \%$ of the dogs in this study showed clinical signs following vaccination, but they were mild and transient in all cases and did not include anaphylactoid reactions. Leptospira vaccines have been said to carry an increased risk of adverse events in puppies younger than 12 weeks of age and in small-breed dogs [32], but a recent study evaluating the incidence rate of owner-reported post-vaccination adverse events found no significant increase in hypersensitivity reactions in dogs vaccinated against Leptospira compared with other vaccines [25]. In the present study, most of the adverse vaccine events included discomfort at the injection site and transient lethargy and inappetence. These are common clinical signs induced by vaccination and have also been reported for other vaccine products [33]. Because additional vaccine components were administered in six of the eleven dogs that showed clinical signs after the first vaccination, the clinical signs could also have been attributable to other vaccine components. However, there was no significant difference in the frequency of adverse events between dogs that did or did not receive other vaccine components at the first or second vaccination. In this study, we decided to administer additional vaccine components as indicated to mimic the situation in the field, where Leptospira vaccines are usually administered together with other vaccine components registered for concurrent application. In light of the high incidence and mortality rate of clinical leptospirosis in dogs in Europe $[4,5,16]$, the benefit of vaccinating dogs with this novel tetravalent vaccine highly outweighs the risk of adverse events following vaccination.

The highest MAT titres after vaccination for the tested serovars were observed for the serovars Canicola, Australis, Grippotyphosa, Bratislava and Autumnalis; the MAT titres were generally low (up to 200) for the vaccine serovar Copenhageni (serogroup Icterohaemorrhagiae), with only $42 \%$ of the study population showing positive titres after the second vaccination. One recent study evaluated vaccine-associated Leptospira antibodies in client-owned dogs following vaccination with four different tetravalent Leptospira vaccines (all containing the serovars Canicola, Grippotyphosa, Icterohaemorrhagiae and Pomona) [10]. The authors found a comparable percentage of dogs with positive titres for the serovars Canicola (100\%), Grippotyphosa $(72 \%)$ and Bratislava (44\%) after vaccination as in the present study, but in contrast to our results, $94 \%$ of the dogs developed positive titres to the serovar Icterohaemorrhagiae. The four vaccines tested in the previous study were different from the vaccine used in the present study; different vaccines could vary in their potential to induce seroconversion to vaccine serovars depending on the strains used in the vaccines. Alternatively, natural 
exposure or differences in immune status could have affected the vaccine responses of individual dogs in our study. Accordingly, we observed highly variable vaccine responses among individual dogs, as also reported in previous studies [10]. It could be argued that we missed the maximal MAT titres against the Icterohaemorrhagiae serogroup, because we sampled the dogs only once 16-31 days after the second immunization, whereas a previous study followed the MAT titres for several weeks following immunization [10]. However, Martin et al. found the highest MAT titres for the vaccine serovars at weeks 4 and 7 after the start of immunization, which is similar to the time point used in the present study to evaluate seroconversion following vaccination. Importantly, a lack of seroconversion does not imply lack of immune protection against the vaccine serovar Copenhageni. Evaluating the protective potential of Leptospira vaccines by use of MAT titres does not provide the entire picture, because previous studies have shown that post-vaccination titres may not correlate with protection [11, 34].

Seroconversion following vaccination was also observed for some non-vaccine serovars, including Autumnalis, Pomona and Pyrogenes. This finding is not unexpected and can be explained by cross-reactivity in MAT among different Leptospira serovars. MAT titres are not serovarspecific, and also in recent studies, dogs developed positive titres against non-vaccine serovars following vaccination with bi- and tetravalent Leptospira vaccines [10-12]. In one study, dogs showed even highest titres to nonvaccine serovars [12]. In the present study, one dog showed maximal seroconversion for the non-vaccine serovar Autumnalis following vaccination, with a titre of 3200 in MAT, although the titres for all of the vaccine serovars were negative at that time except for the serovar Canicola (800). Besides vaccination, natural exposure to the serovar Autumnalis coinciding with vaccination could have accounted for seroconversion in this animal. However, the dog showed no clinical signs or abnormalities in haematology and blood biochemistry compatible with leptospirosis at that time. Our results indicate that even high titres $(\geq 800)$ against non-vaccine serovars should be interpreted with caution in vaccinated dogs exhibiting signs consistent with leptospirosis.

Half of the dogs showed positive MAT titres at study enrolment for the serovar Canicola, although vaccines containing this serovar had been administered to the dogs up to 1745 days prior to sampling. The dog is assumed to be a natural reservoir host of $L$. interrogans serovar Canicola, and before the introduction of efficient vaccines, clinical leptospirosis in dogs has commonly been associated with this serovar. Because the present study was performed in privately owned dogs with outdoor access, natural exposure to leptospires in addition to vaccination could have resulted in antigenic stimulation in these dogs. Our results are in contrast to an earlier study that evaluated Leptospira antibodies in privately owned dogs in Switzerland between 1991 and 1996 [35]. In that study, $34 \%$ of the dogs showed positive Leptospira antibody titres, with seroconversion to other serovars, e.g., Bratislava, Australis, Icterohaemorrhagiae, Pomona and Grippotyphosa, being common. Moreover, in a survey of 259 dogs from Switzerland over a 10-year-period before the introduction of the tetravalent vaccine, only $12.3 \%$ of the dogs showed seropositivity to serovar Canicola [5]. We cannot conclude from the present data whether the positive MAT titres to Canicola were due to previous vaccination or natural exposure to this serovar. However, in light of the large time span elapsed since the last vaccination in some of the seropositive dogs, natural exposure seems likely, and vaccination against this serovar can still be advocated.

In the present study, dogs were specifically examined for evidence of myocarditis due to anecdotal reports of cardiac changes after vaccination with this new vaccine. There is some credibility to such a potential association, because natural infection with leptospirosis may, indeed, cause myocarditis. Even though clinically important myocarditis is rare in dogs and people after natural infection $[6,36]$, subclinical myocarditis may be quite commonly present in both species based on biochemical abnormalities, specifically the elevation of cTroponin I [6], and histopathological findings [7]. Furthermore, myocarditis has been documented as an adverse event following vaccination in humans, such as after smallpox vaccination [37]. The present study found no echocardiographic, electrocardiographic or biochemical evidence of subclinical vaccine-induced myocarditis. Based on the limited number of dogs included in the present study, myocarditis as a rare complication of leptospirosis vaccination cannot, however, completely be ruled out.

The present study has some limitations. Due to the prospective study design, the number of dogs included in the study was too small to evaluate rare adverse events induced by the vaccine. This could be assessed by large retrospective studies and pharmacovigilance data. The present study also did not follow the course of the MAT titres after vaccination and therefore cannot evaluate the time point of maximal MAT titres and the duration of seropositivity induced by the vaccine. Third, the inclusion of other vaccine components could have influenced the clinical and serological response of the dogs. However, we chose this setting to most accurately mimic the situation in the field, where Leptospira vaccines are commonly administered together with other vaccine components licenced for concurrent administration. Finally, measurements of cTroponin I were only obtained before the first and 16-31 days after the second vaccination. It 
can be argued that an elevation might have been present earlier after vaccination. However, the complete absence of any evidence of morphological, functional and electrical cardiac abnormalities makes clinically important myocardial damage very unlikely.

\section{Conclusions}

The present study indicates that the novel tetravalent Leptospira vaccine Nobivac ${ }^{\ominus}$ L4 is generally welltolerated and that clinical signs following vaccination were mild and transient. Seroconversion following vaccination differed considerably among individual dogs and among the vaccine serovars. High titres to nonvaccine serovars were present in some dogs following vaccination, which hampers the interpretation of single MAT titres in vaccinated dogs with clinical signs consistent with leptospirosis. In the light of the high incidence and mortality rate associated with clinical leptospirosis in dogs in Europe, vaccination with this tetravalent vaccine can be advocated for every dog with potential exposure to leptospires.

\section{Additional files}

Additional file 1: Medical history and clinical examination findings at different time points before and after vaccination. (DOCX $37 \mathrm{~kb}$ )

Additional file 2: Selected haematology results of the dogs before and after vaccination. Values outside the reference range are shown in bold, significant differences between $\mathrm{T} 0$ and $\mathrm{T} 2$ are shown in italics. (DOCX $35 \mathrm{~kb}$ )

Additional file 3: Selected blood biochemistry results before and after vaccination. Values outside the reference range are shown in bold, significant differences between T0 and T2 are shown in italics. (DOCX $35 \mathrm{~kb}$ )

\section{Abbreviations}

ALAT: Alanine aminotransferase; AP: Alkaline phosphatase; Cl: Confidence interval; cTroponin I: Cardiac Troponin I; \%FS: Fractional shortening (\%); MAT: Microscopic agglutination test; LA/Ao: Left atrial to aortic ratio; LVDd: Left ventricular diameter in diastole; LVDs: Left ventricular diameter in systole; OIE: Office International des Epizooties; $p_{F}$ : $p$-value of Fisher's exact test; $p_{w}$ : $p$-value of Wilcoxon signed rank test; $r_{\text {sp }}$ : Correlation coefficient of Spearman rank correlation test; T0: Before first vaccination; T1: Second vaccination 20-28 days after the first vaccination; T2: 41-54 days after the first and 16-31 days after the second vaccination; T3: 368-434 days after the first vaccination and $347-413$ days after the second vaccination (1 year booster)

\section{Acknowledgements}

The authors thank the technicians of the Clinical Laboratory for their excellent laboratory assistance and Stefanie Müller from the Institute of Veterinary Bacteriology for excellent technical assistance.

\section{Funding}

AS was supported by a research grant (Forschungskredit, FK-53210-01-01) from the University of Zurich. The funding body played no role in the design of the study and collection, analysis, and interpretation of data and in writing the manuscript.

\section{Availability of data and materials}

The datasets used and/or analysed during the current study are available from the corresponding author on reasonable request.

\section{Authors' contributions}

BW, RHL and CR conceived the study. BW and AS were responsible for the study coordination and the data and sample collection. SRC was responsible for the serological analyses by MAT. BR was responsible for the haematology and blood biochemistry tests. JM and TG performed the echocardiography examinations. BW drafted the manuscript. BW, AS, CR and RHL edited the manuscript. All of the authors read and approved the final manuscript.

\section{Competing interests}

The authors declare that they have no competing interests.

\section{Ethics approval and consent to participate}

Written informed consent was obtained from all of the owners of the dogs participating in this study, and the study was performed in adherence to a high standard (best practice) of veterinary care. All experiments were performed according to Swiss law and sample collections were officially approved by the veterinary office of the canton of Zurich (TVB 72/11 and TVB 042/15).

\section{Publisher's Note}

Springer Nature remains neutral with regard to jurisdictional claims in published maps and institutional affiliations.

\section{Author details}

${ }^{1}$ Clinical Laboratory, Vetsuisse Faculty, University of Zurich, Winterthurerstr. 260, 8057 Zurich, Switzerland. ${ }^{2}$ Center for Clinical Studies, Vetsuisse Faculty, University of Zurich, Winterthurerstr. 260, 8057 Zurich, Switzerland. ${ }^{3}$ Clinic for Small Animal Internal Medicine, Vetsuisse Faculty, University of Zurich, Winterthurerstr. 260, 8057 Zurich, Switzerland. ${ }^{4}$ Institute of Veterinary Bacteriology, Vetsuisse Faculty, University of Bern, Länggassstr. 122, 3001 Bern, Switzerland.

Received: 31 January 2017 Accepted: 11 May 2017

Published online: 25 May 2017

\section{References}

1. Sykes JE, Hartmann K, Lunn KF, Moore GE, Stoddard RA, Goldstein RE. 2010 ACVIM small animal consensus statement on leptospirosis: diagnosis, epidemiology, treatment, and prevention. J Vet Intern Med. 2011;25(1):1-13. doi:10.1111/j.1939-1676.2010.0654.x.

2. Schuller S, Francey T, Hartmann K, Hugonnard M, Kohn B, Nally JE, et al. European consensus statement on leptospirosis in dogs and cats. J Small Anim Pract. 2015:56(3):159-79. doi:10.1111/jsap.12328.

3. Alexander AD, Evans LB, Baker MF, Baker HJ, Ellison D, Marriapan M. Pathogenic leptospiras isolated from Malaysian surface waters. Appl Microbiol. 1975;29(1):30-3.

4. Ellis WA. Control of canine leptospirosis in Europe: time for a change? Vet Rec. 2010;167(16):602-5. doi:10.1136/vr.c4965.

5. Major A, Schweighauser A, Francey T. Increasing incidence of canine leptospirosis in Switzerland. Int J Environ Res Public Health. 2014;11 (7):7242-60. doi:10.3390/ijerph110707242.

6. Mastrorilli C, Dondi F, Agnoli C, Turba ME, Vezzali E, Gentilini F. Clinicopathologic features and outcome predictors of Leptospira interrogans Australis serogroup infection in dogs: a retrospective study of 20 cases (2001-2004). J Vet Intern Med. 2007;21(1):3-10.

7. Shah K, Amonkar GP, Kamat RN, Deshpande JR. Cardiac findings in leptospirosis J Clin Pathol. 2010;63(2):119-23. doi:10.1136/jcp.2009.069575.

8. Levett PN. Leptospirosis. Clin Microbiol Rev. 2001;14(2):296-326. doi:10. 1128/CMR.14.2.296-326.2001

9. Goris MG, Hartskeerl RA. Leptospirosis serodiagnosis by the microscopic agglutination test. Curr Protoc Microbiol. 2014;32:Unit 12E 5. doi:10.1002/ 9780471729259.mc12e05s32.

10. Martin LE, Wiggans KT, Wennogle SA, Curtis K, Chandrashekar R, Lappin MR. Vaccine-associated Leptospira antibodies in client-owned dogs. J Vet Intern Med. 2014:28(3):789-92. doi:10.1111/jvim.12337.

11. Barr SC, McDonough PL, Scipioni-Ball RL, Starr JK. Serologic responses of dogs given a commercial vaccine against Leptospira interrogans serovar pomona and Leptospira kirschneri serovar grippotyphosa. Am J Vet Res. 2005;66(10):1780-4.

12. Midence JN, Leutenegger CM, Chandler AM, Goldstein RE. Effects of recent Leptospira vaccination on whole blood real-time PCR testing in healthy client-owned dogs. J Vet Intern Med. 2012;26(1):149-52. doi:10.1111/j.1939-1676.2011.00852.x. 
13. Greenlee JJ, Alt DP, Bolin CA, Zuerner RL, Andreasen CB. Experimental canine leptospirosis caused by Leptospira interrogans serovars pomona and bratislava. Am J Vet Res. 2005;66(10):1816-22.

14. Fraune $C K$, Schweighauser A, Francey $T$. Evaluation of the diagnostic value of serologic microagglutination testing and a polymerase chain reaction assay for diagnosis of acute leptospirosis in dogs in a referral center. J Am Vet Med Assoc. 2013;242(10):1373-80. doi:10.2460/javma.242.10.1373.

15. Jull DJ, Heath KR. The evaluation of a combined $L$. canicola and $L$. icterohaemorrhagiae vaccine on hamsters and dogs. J Small Anim Pract. 1961:1:245-58.

16. Kohn B, Steinicke K, Arndt G, Gruber AD, Guerra B, Jansen A, et al. Pulmonary abnormalities in dogs with leptospirosis. J Vet Intern Med. 2010;24(6):1277-82. doi:10.1111/j.1939-1676.2010.0585.x.

17. Sonrier C, Branger C, Michel V, Ruvoen-Clouet N, Ganiere JP, Andre-Fontaine G. Evidence of cross-protection within Leptospira interrogans in an experimental model. Vaccine. 2000;19(1):86-94.

18. Plesko I, Lataste-Dorolle C. Intertype immunity relations of leptospira strains belonging to the 'Australia' serogroup. Biologia. 1970;25:403-11.

19. Adler B, Faine $\mathrm{S}$. The antibodies involved in the human immune response to leptospiral infection. J Med Microbiol. 1978;11(4):387-400. doi:10.1099/ 00222615-11-4-387.

20. Klaasen $\mathrm{HL}$, van der Veen M, Molkenboer MJ, Sutton D. A novel tetravalent Leptospira bacterin protects against infection and shedding following challenge in dogs. Vet Rec. 2013;172(7):181. doi:10.1136/vr.101100.

21. Klaasen $\mathrm{HL}$, van der Veen $\mathrm{M}$, Sutton $\mathrm{D}$, Molkenboer MJ. A new tetravalent canine leptospirosis vaccine provides at least 12 months immunity against infection. Vet Immunol Immunopathol. 2014;158(1-2):26-9. doi:10.1016/j.vetimm.2013.08.002.

22. Andre-Fontaine G. Diagnosis algorithm for leptospirosis in dogs: disease and vaccination effects on the serological results. Vet Rec. 2013;172(19):502. doi:10.1136/vr.101333.

23. Hoffmann A, Schwedinger E, Werner G, Cussler K. Pharmakovigilanzreport Tierimpfstoffe. Deutsches Tierärzteblatt. 2016:8:1176-8.

24. Müntener CR, Bruckner L, Kupper J, Althaus FR, Schäublin M. Vigilance der Tierarzneimittel: Gemeldete unerwünschte Wirkungen im Jahr 2013. Schweiz Arch Tierheilkd. 2014:156(11):519-25.

25. Yao PJ, Stephenson N, Foley JE, Toussieng CR, Farver TB, Sykes JE, et al. Incidence rates and risk factors for owner-reported adverse events following vaccination of dogs that did or did not receive a Leptospira vaccine. J Am Vet Med Assoc. 2015;247(10):1139-45. doi:10.2460/javma.247.10.1139.

26. Moore GE, Guptill LF, Ward MP, Glickman NW, Faunt KK, Lewis HB, et al. Adverse events diagnosed within three days of vaccine administration in dogs. J Am Vet Med Assoc. 2005;227(7):1102-8.

27. Weissenbacher S, Riond B, Hofmann-Lehmann R, Lutz H. Evaluation of a novel haematology analyser for use with feline blood. Vet J. 2011;187(3):381-7. doi:10.1016/j.tvjl.2010.01.005

28. Gallay-Lepoutre J, Belanger MC, Nadeau ME. Prospective evaluation of Doppler echocardiography, tissue Doppler imaging and biomarkers measurement for the detection of doxorubicin-induced cardiotoxicity in dogs: a pilot study. Res Vet Sci. 2016;105:153-9. doi:10.1016/j.rvsc.2016.02.001.

29. Office International des Epizooties (OIE). Chapter 2.1.9. Leptospirosis. In: Terrestrial Manual. 7th ed; 2012. p. 251-64.

30. Thomas WP, Gaber CE, Jacobs GJ, Kaplan PM, Lombard CW, Moise NS, et al. Recommendations for standards in transthoracic two-dimensional echocardiography in the dog and cat. Echocardiography Committee of the Specialty of cardiology, American College of Veterinary Internal Medicine. J Vet Intern Med. 1993;7(4):247-52.

31. Rishniw M, Erb HN. Evaluation of four 2-dimensional echocardiographic methods of assessing left atrial size in dogs. J Vet Intern Med. 2000;14(4):429-35.

32. Paul MA, Appel M, Barrett R, Carmichael LE, Childers H, Cotter S, et al. Report of the American animal hospital association (AAHA) canine vaccine task force: executive summary and 2003 canine vaccine guidelines and recommendations. J Am Anim Hosp Assoc. 2003;39(2):119-31.

33. Day MJ. Vaccine side effects: fact and fiction. Vet Microbiol. 2006;117:51-8.

34. Minke JM, Bey R, Tronel JP, Latour S, Colombet G, Yvorel J, et al. Onset and duration of protective immunity against clinical disease and renal carriage in dogs provided by a bi-valent inactivated leptospirosis vaccine. Vet Microbiol. 2009;137(1-2):137-45. doi:10.1016/j.vetmic.2008.12.021.

35. Steger-Lieb A, Gerber B, Nicolet J, Gaschen F. An old disease with a new face: canine leptospirosis does not lose its relevance. Schweiz Arch Tierheilkd. 1999;141(11):499-507.
36. Abgueguen P, Delbos V, Blanvillain J, Chennebault JM, Cottin J, Fanello S, et al. Clinical aspects and prognostic factors of leptospirosis in adults. Retrospective study in France. J Inf Secur. 2008;57(3):171-8. doi:10.1016/ j.jinf.2008.06.010.

37. Engler RJ, Nelson MR, Collins LC Jr, Spooner C, Hemann BA, Gibbs BT, et al. A prospective study of the incidence of myocarditis/pericarditis and new onset cardiac symptoms following smallpox and influenza vaccination. PLoS One. 2015;10(3):e0118283. doi:10.1371/journal.pone.0118283.

\section{Submit your next manuscript to BioMed Central and we will help you at every step:}

- We accept pre-submission inquiries

- Our selector tool helps you to find the most relevant journal

- We provide round the clock customer support

- Convenient online submission

- Thorough peer review

- Inclusion in PubMed and all major indexing services

- Maximum visibility for your research

Submit your manuscript at www.biomedcentral.com/submit
) Biomed Central 